\title{
Infiuencia del apoyo educativo en Ia disminución del tabaquismo en alumnos de una escuela secundaria
}

\author{
Valdés-Frausto Cynthia Guadalupe*, Rodríguez-Ramírez José Manuel**, \\ Casillas-Casillas Elizabeth***, Salas-Sánchez Ana Berenice ${ }^{* * *}$, Hernández-Delgadillo Nicolás \\ Alejandro****, Gómez-Cardona Juan Pablo**
}

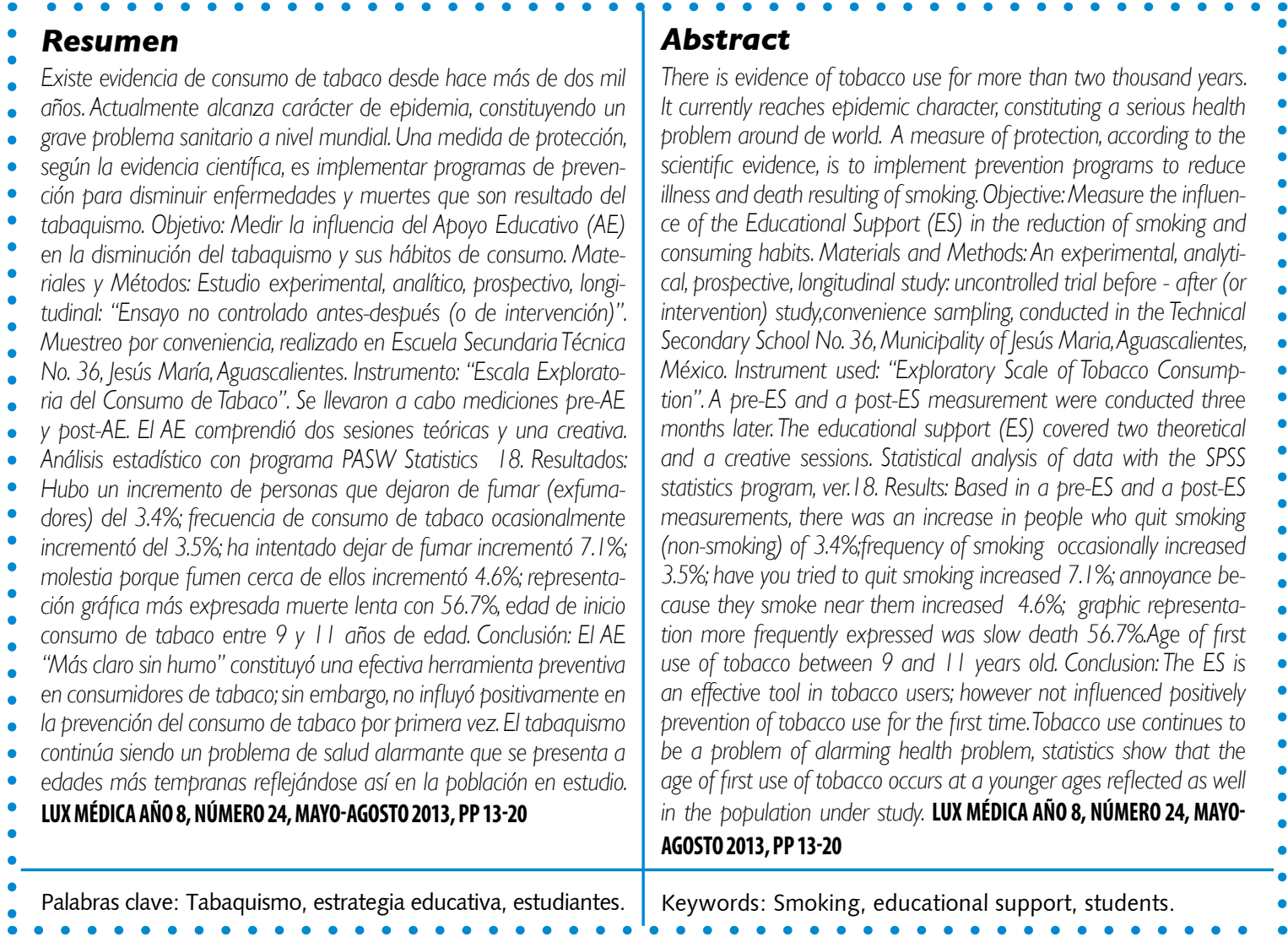

Enfermera General "C" del Hospital Regional de Alta Especialidad del Bajío

** Profesores del Departamento Enfermería del Centro de Ciencias de la Salud de la Universidad Autónoma de Aguascalientes

*** Profesora investigadora del Departamento de Optometría del Centro de Ciencias de la Salud de la Universidad Autónoma de Aguascalientes

**** Profesora del Departamento de Salud Pública del Centro de Ciencias de la Salud de la Universidad Autónoma de Aguascalientes.

Fecha de recibido: 11 de junio 2013

Fecha de aceptación 1 de agosto de 2013

Correspondencia MCE Juan Pablo Gómez Cardona Departamento de Enfermería del Centro de Ciencias de la Salud de la Universidad Autónoma de Aguascalientes. Av. Universidad 940 Ciudad Universitaria Edificio 105. CP 20131, Aguascalientes, Ags., México. Teléfono 014499108437 correo electrónicojpgomezc@correo.uaa.mx 


\section{Introducción}

El consumo de tabaco se inicia desde hace más de dos mil años, pero en el siglo XX alcanza carácter de epidemia y se constituye en un grave problema sanitario a nivel mundial, traspasando fronteras, sin respetar razas, ni culturas, afectando a todos los grupos sociales, sin limitaciones de género, edad, estrato social o religión. ${ }^{1}$ En total, la epidemia de tabaquismo mata cada año a casi 6 millones de personas, de proseguir esta tendencia, en el año 2030 la cifra aumentará a más de 8 millones anuales. ${ }^{2}$

La Organización Mundial de la Salud (OMS), considera al tabaquismo como la principal causa evitable de discapacidad, enfermedad y muerte prematura. ${ }^{1}$ El consumo de tabaco es un factor de riesgo para seis de las ocho principales causas de defunción en el mundo. ${ }^{1}$ En México, el tabaquismo se considera un problema prioritario de salud ya que diariamente fallecen 165 personas por causas atribuibles al tabaco y anualmente mueren $60,000 .^{2}$ Uno de cada dos fumadores que comienzan a fumar en edad temprana y continúa haciéndolo durante toda su vida de forma regular, morirá probablemente a mediana edad, perdiendo alrededor de 22 años de vida saludable. ${ }^{1}$

El tabaquismo, acarrea consigo graves consecuencias sociales como: deficiente calidad de vida, desnutrición, ausentismo laboral y escolar en los niños y adolescentes al ser fumadores activos o pasivos. $^{3}$ El tabaco al ser una droga legal y de inicio, que funciona como antecesor para otras drogas, ${ }^{4}$ es un producto que se encuentra a la mano en cualquier sitio, a cualquier hora del día y la noche, su venta se encuentra desde expendios, hasta máquinas automáticas, bares, restaurantes y supermercados. La Encuesta Nacional de Adicciones 2011 (ENA 2011), reportó que los fumadores activos entre 12 y 17 años de edad compraron sus cigarros en tiendas de abarrotes, vendedores ambulantes o en tianguis. ${ }^{5}$

Los programas de mercadotecnia de la industria tabacalera están basados en la promoción de los diversos productos tabáquicos que ofrecen: masticables, inhalados, chupados o esnifados, promoviéndolo como un producto símbolo de moda y madurez, no dañino para la salud; su principal objetivo, es el consumo de tabaco por niños, adolescentes, aprovechando la vulnerabilidad de la edad para ser aceptados por un grupo en la sociedad, aunque esto signifique la práctica de un hábito no saludable. Según la ENA 2011, la edad promedio de consumo de tabaco, comienza a los 14.1 años de edad ${ }^{5}$ y se presenta a edades cada vez más tempranas.

En la Teoría del Déficit del Autocuidado de Dorothea Elizabeth Orem, se describe la Teoría de los Sistemas de Enfermería, la cual explica la manera en que las enfermeras/os pueden atender a los individuos $^{6}$, el Sistema de Enfermería de Apoyo-Educación utilizado cuando la enfermera actúa ayudando a los individuos para que sean capaces de realizar las actividades de autocuidado ${ }^{7}$, comprende cinco métodos de asistencia, uno de los cuales es el de enseñar a otro ${ }^{8}$, utilizado para aquellas personas que necesitan desarrollar sus conoci- 
mientos y habilidades particulares. ${ }^{9}$ El empleo de este método requiere que la enfermera conozca con precisión lo que la persona ayudada necesita saber y utilizar los métodos de enseñanza apropiados. ${ }^{10}$

De acuerdo a la evidencia científica disponible ${ }^{11}$ y a diversas recomendaciones internacionales, la implementación de estrategias educativas representa una medida de protección. ${ }^{12}$ La educación es una de las principales estrategias aprobadas por las distintas organizaciones tanto a nivel mundial ${ }^{13}$, nacional y estatal, incluida ésta en los diferentes programas que apoyan la prevención, disminución de enfermedades y muertes producidas por el consumo de tabaco. Muchos adolescentes son incapaces de identificar los problemas que el tabaquismo conlleva, es importante implementar en instituciones educativas, un programa orientado a proporcionar información sobre consecuencias y riesgos del tabaco, de manera preventiva y correctiva.

La investigación, tuvo como objetivo medir la influencia del Apoyo Educativo $(A E)$ en los hábitos y la disminución del consumo de tabaco entre los estudiantes.

| | | | | | | | | | | | | | | | | | | | | | | | | | | | | | | | | | | | | | | | | | | | | | | | | | | | | | | | | | | | | | | | | | | | | | | | | | | | | | | | | | | | | | | | | | | | | | | | | | | | | | | | | |

\section{Material y métodos}

Estudio experimental, analítico, prospectivo, longitudinal: "Ensayo no controlado antes-después (o de intervención)"14. Muestreo por conveniencia conformado por la totalidad de alumnos del primer grado, turno matutino $(n=213)$, de la Escuela Secundaria Técnica No. 36 (EST No. 36), Municipio de Jesús María, Aguascalientes. La investigación se llevó a cabo entre los meses de noviembre 2012 a febrero 2013.

El instrumento utilizado fue la "Escala Exploratoria del Consumo de Tabaco" (EECT), la adaptación mexicana del instrumento (tropicalización), obtuvo un coeficiente de consistencia interna de 0.97 (15). La EECT consta de 3 partes: la primera integrada por 4 ítems de identificación del estudiante (edad, grado, ocupación y sexo); la segunda constituye un extracto de la ENA 2011 ${ }^{6}$, integrada por 9 ítems que proporcionan datos de prevalencia actual y experimentación o uso regular de tabaco, edad de inicio, disposición para dejar de fumar y la exposición pasiva/activa al humo de tabaco ajeno; la tercer parte integrada por 6 ítems de tipo opción múltiple, se trata del test Fagerstrom ${ }^{15}$ utilizado de manera internacional y por el Consejo Nacional contra las Adicciones (CONADIC), que analiza la cantidad de cigarrillos consumidos, la compulsión por fumar y la dependencia nicotínica.

El abordaje de la población en estudio se dividió en tres etapas: en la primera se aplicó el cuestionario EECT (medición pre-AE); durante la segunda etapa se implementó la estrategia de AE titulada "Más Claro sin Humo", la cual consistió en dos sesiones teóricas con la siguiente temática: a)Daños a la salud provocados por el tabaquismo, b) Mercadotecnia, c)Costos, d)Composición del cigarrillo y e)Beneficios por dejar de fumar y una sesión creativa con la finalidad de expresar por medio de carteles lo trabajado en las sesiones teóricas; la tercera llevada a cabo tres meses posteriores a la implementación del $A E$, aplicando el cuestionario EECT (medición post-AE).

Se llevó a cabo estadística descriptiva (porcentajes, frecuencias y desviación estándar e inferencial, razón de momios (RM) con ayuda del programa PASW Statistics versión 18. 


\section{Resultados}

Se estudiaron 213 alumnos inscritos en el primer año de la EST No. 36 del Municipio de Jesús María, Aguascalientes. En la tabla 1 se describen las variables sociodemográficas; el rango de edad fue de 11 a 15 años con una media de 12.04 años; el sexo predominante fue el femenino con un $54.5 \%$, la ocupación laboral tuvo un incremento del $13.6 \%$, pasando del $27.2 \%$ al $40.8 \%$ entre la medición pre y post-AE.

La influencia del AE "Más claro sin humo", tuvo un efecto positivo apreciándose en las siguientes variables: personas que dejaron de fumar (ex-fumadores) incrementó $3.5 \%$ pasando del $15 \%$ al $18.5 \%$, frecuencia de consumo de tabaco: ocasionalmente incrementó 3.5\% pasando del $9.9 \%$ al $13.4 \%$, ha intentado dejar de fumar incrementó $7.1 \%$ pasando del $17.8 \%$ al $24.9 \%$, lugar de convivencia casa disminuyó $5.1 \%$ pasando del $56.3 \%$ al $51.2 \%$, molestia porque fumen cerca de ellos incrementó $4.6 \%$ pasando del $77.6 \%$ al $82.2 \%$, según mediciones pre y post $A E$ respectivamente (tabla 1). Sin embargo en el grupo de fumadores activos el efecto del $A E$ fue negativo, ya que se incrementó $5 \%$ pasando del $8.5 \%$ al $13.5 \%$, apreciándose también en la variable prevalencia de consumo de tabaco en su dimensión alguna vez en la vida incrementando un $11.8 \%$ pasando del $23.5 \%$ al $35.3 \%$ (tabla 1 ).

En la tercer parte del cuestionario integrada por algunos de los ítems test Fagerstrom ${ }^{15}$ los patrones de consumo: cantidad de consumo de tabaco diario, consumo de tabaco durante el día, tiempo de consumo el primer cigarrillo después de levantarse, cigarrillo que menos dejaría pasar durante el día, consumo de tabaco en lugares prohibidos y consumo de tabaco durante enfermedad, en su dimensión no fumo diario mostraron un incremento porcentual positivo posterior al AE (tabla 1).

\section{Tabla |}

\section{Patrones de Consumo de Tabaco}

\begin{tabular}{|c|c|c|c|}
\hline Variables & & Porcentaje Pre-AE & Porcentaje Post-AE \\
\hline $\begin{array}{l}\text { Distribución de la población estudiada } \\
\text { según consumo de tabaco }\end{array}$ & $\begin{array}{l}\text { Fumadores Activos } \\
\text { Ex Fumadores } \\
\text { Nunca Fumadores }\end{array}$ & $\begin{array}{c}8.5 \\
15 \\
76.5 \\
\end{array}$ & $\begin{array}{l}16.9 \\
18.4 \\
64.7 \\
\end{array}$ \\
\hline Prevalencia de consumo de tabaco & $\begin{array}{l}\text { Alguna vez en la vida } \\
\text { En el último año } \\
\text { En el último mes }\end{array}$ & $\begin{array}{l}23.5 \\
8.5 \\
37.1\end{array}$ & $\begin{array}{l}35.3 \\
16.9 \\
43.3 \\
\end{array}$ \\
\hline Frecuencia de consumo de tabaco & $\begin{array}{l}\text { Semanalmente } \\
\text { Ocasionalmente }\end{array}$ & $\begin{array}{l}2.3 \\
9.9\end{array}$ & $\begin{array}{c}1.5 \\
13.4\end{array}$ \\
\hline Ha intentado dejar de fumar & $\mathrm{Si}$ & 17.8 & 24.9 \\
\hline $\begin{array}{l}\text { Lugar de convivencia de } 1 \text { a } 5 \text { personas } \\
\text { fumadoras }\end{array}$ & $\begin{array}{l}\text { Casa } \\
\text { Clase } \\
\text { Trabajo }\end{array}$ & $\begin{array}{c}56.3 \\
6.6 \\
7.5\end{array}$ & $\begin{array}{c}51.2 \\
7.5 \\
3\end{array}$ \\
\hline Molestia porque fumen cerca de ellos & $\mathrm{Si}$ & 77.6 & 82.2 \\
\hline Cantidad de consumo de tabaco diario & No fumo diario & 24.9 & 27.9 \\
\hline Consumo de tabaco durante el día & $\begin{array}{l}\text { Mañana } \\
\text { No fumo diario }\end{array}$ & $\begin{array}{l}0.5 \\
16\end{array}$ & $\begin{array}{r}1.5 \\
23.9 \\
\end{array}$ \\
\hline $\begin{array}{l}\text { Tiempo de consumo del primer } \\
\text { cigarrillo después de levantarse }\end{array}$ & No fumo diario & 20.7 & 25.4 \\
\hline $\begin{array}{l}\text { Cigarrillo que menos dejaría pasar } \\
\text { durante el día }\end{array}$ & No fumo diario & 18.8 & 2.9 \\
\hline Consumo de tabaco en lugares prohibidos & $\begin{array}{l}\mathrm{Si} \\
\text { No fumo diario }\end{array}$ & $\begin{array}{c}1.4 \\
13.6 \\
\end{array}$ & $\begin{array}{c}4.5 \\
15.9 \\
\end{array}$ \\
\hline Consumo de tabaco durante la enfermedad & No & 23.5 & 32.8 \\
\hline
\end{tabular}


Como resultado de la sesión creativa, las siete representaciones gráficas más sobresalientes de los carteles titulados "Más Claro sin Humo" fueron: muerte lenta, droga- tóxico- legal, diente amarillo/ sabor amargo, enfermedades del corazón, enfer- medades respiratorias, cabello seco y desventajas por su costo y por último tipos de Fumadores y cáncer (tabla 2). Es importante resaltar que en cada cartel se expresaron dos o más representaciones gráficas.

\section{Tabla 2}

\section{Resultados de la sesión creativa (representación gráfica)}

\begin{tabular}{lc|}
\hline Representación gráfica & $\%$ \\
\hline Muerte lenta & 56.7 \\
\hline Droga- tóxico- legal & 54 \\
\hline Diente amarillo / sabor amargo & 32.4 \\
\hline Enfermedades del corazón & 29.7 \\
\hline Enfermedades respiratorias & 27 \\
\hline Cabello seco y desventajas por su costo & 18 \\
\hline Tipos de fumadores y cáncer & 13.5 \\
\hline
\end{tabular}

En las variable intentar dejar de fumar, te molesta que fumen cerca de ti y abstenerse de fumar en lugares públicos, en los últimos 30 días pre- $A E$, representan una diferencia positiva de OR 2.4 con $p<$ 0.03 , una diferencia negativa de OR 0.22 y una diferencia positiva de 9.2 respectivamente; la variable sexo femenino, independiente de la temporalidad del estudio, se mantiene como una constante con un OR 1.4 (tabla 9.4).

\section{Tabla 3}

\section{Inferencias en variables de la EECT (últimos 30 días) * $\mathbf{p}<0.05$}

\begin{tabular}{|lcccc|}
\hline Variable & & RM & IC & P \\
\hline Intentar dejar de fumar & Pre & 3.9 & $1.14-13.7$ & $0.03^{*}$ \\
& Post & 1.46 & $0.47-4.47$ & 0.34 \\
\hline Te molesta que fumen cerca de ti & Pre & 0.05 & $0.14-2.21$ & 0.30 \\
& Post & 0.27 & $0.9-0.79$ & 0.1 \\
\hline Sexo Femenino & Pre & 1.49 & $0.42-5.2$ & 0.37 \\
& Post & 1.4 & $0.32-6.06$ & 0.64 \\
\hline Abstenerse de fumar en lugares públicos & Pre & 10 & $0.83-119.7$ & 0.14 \\
& Post & 0.71 & $0.22-2.44$ & 0.62 \\
\hline
\end{tabular}

La gráfica 1 muestra que del total de la población estudiada, la edad promedio de inicio al consumo de tabaco fue entre los
9 y 11 años de edad, el 17\% de los estudiantes. 


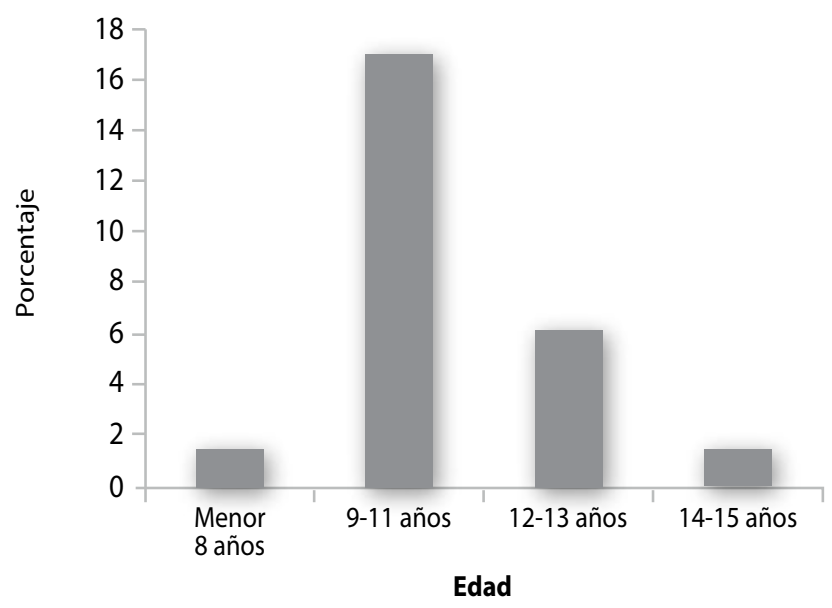

Gráfica 1: Distribución de la edad de inicio de consumo de tabaco entre los estudiantes evaluados

\section{Discusión}

De acuerdo con la ENA $2011^{5}$ el $12.3 \%$ de la población de adolescentes de entre 12 y 17 años de edad son fumadores activos, 9.9\% corresponde a ex-fumadores y $77.8 \%$ nunca ha fumado tabaco, el porcentaje es más bajo con los resultados de la EECT aplicada en la EST No. 36, donde el $8.5 \%$ corresponden a fumadores activos, $15 \%$ son ex-fumadores y el $76.5 \%$ nunca ha fumado tabaco en la medición pre$A E$, mientras que en la medición post- $A E$ incrementó a un $16.9 \%, 18.4 \%$ y $64.7 \%$ respectivamente; podemos resaltar que el impacto del $A E$ fue positivo ya que el porcentaje de ex-fumadores, es decir las personas que dejaron de fumar, aumentó en la medición post-AE (tabla 1).

En la variable frecuencia de consumo de tabaco en la dimensión ocasionalmente, el incremento observado entre la medición pre-AE y post-AE sugiere que el adolescente aún no posee un patrón de consumo del tipo adictivo sino ocasional.

Respecto al lugar de convivencia de 1-5 personas fumadoras, podemos resaltar que la dimensión casa, es considerado un factor de riesgo y no de protección por presentar el porcentaje más alto de convivencia con fumadores, en la medición pre-AE con un
$56.3 \%$ y un $51.2 \%$ posterior al $A E$, comparado con las dimensiones de clase con el $6.6 \%$ y el $7.5 \%$, y trabajo con el $7.5 \%$ $y$ el $3 \%$ en las mediciones pre-AE y post$A E$ respectivamente, lo anterior coincide con el Instituto Nacional de Estadística Geografía e Informática (INEGI) "Estadísticas a propósito del Día mundial sin tabaco"$^{16}$ que afirma que el principal lugar de exposición al humo de tabaco es el hogar (tabla 1).

Con base en el análisis de la tabla 1, diversos patrones de consumo en su dimensión no fumo diario, mostraron un incremento entre la medición pre y post$A E$, refirmando que el consumo de tabaco de tipo ocasional, tal vez es atribuible a la compañía y reuniones del grupo de amigos.

Diversos estudios han encontrado que la edad promedio de consumo de tabaco por primera vez ha oscilado. De acuerdo con la ENA $2008^{18}$ la edad de inicio de consumo de tabaco por primera vez en Aguascalientes fue a los 17 años de edad y las principales causas fueron la curiosidad y la convivencia con fumadores ${ }^{1}$; la ENA 2011, reportó que la edad de inicio de consumo diario de tabaco de los adolescentes de entre 12 y 17 años de edad comienza a los 14.1 años ${ }^{5}$, colocando a la región occiden- 
tal conformada por Aguascalientes, Zacatecas, Jalisco, Colima y Nayarit, como la segunda región con más alto porcentaje de fumadores activos.

Al estudio realizado por Rodríguez-Ramírez y cols. denominado "Prevalencia de consumo de tabaco en estudiantes de bachillerato" en el $2012^{18}$, la edad promedio de consumo de tabaco por primera vez fue a los 14.3 años, difiriendo con este estudio en el que la edad promedio oscila en un intervalo entre los 9 y 11 años de edad (gráfica 1), es decir, a una edad más temprana, se cree que su explicación se vincula a cuatro aspectos principales: a)Curiosidad, b)Alto porcentaje de convivencia con fumadores principalmente en casa, c)Presión de pares, por la necesidad de pertenencia y aceptación a un grupo social, pues el que algunos o la mayoría de sus amigos fume aumenta la probabilidad de ser fumador un $54 \%$, según estudio realizado por Valdés
Salgado y cols. en el 2006 denominado "Los retos del convenio marco para el control del tabaco en México: un diagnóstico a partir de la Encuesta sobre Tabaquismo en Jóvenes" y d)Mercadotecnia de la industria tabacalera dirigida principalmente a este grupo de edad. ${ }^{19}$

Según el estudio realizado por Valdés Salgado y cols., los estudiantes en edad secundaria que NO reconocen al tabaco como una droga, tienen $77 \%$ mayor posibilidad de ser fumadores ${ }^{19}$, por lo que se muestra una influencia positiva del $A E$ ya que el $54 \%$ de los cárteles expresaron este reconocimiento (tabla 2).

Importante resaltar que los estudiantes expresaron los tres principales daños a la salud que provoca el tabaco: enfermedades del corazón, enfermedades respiratorias y cáncer, aunque no las reconocen como las principales anteponiendo: diente amarillo, sabor amargo y el cabello seco (tabla 2).

\section{Conclusiones}

El AE "Más claro sin humo" constituyó una efectiva herramienta preventiva en consumidores de tabaco; sin embargo, no influyó positivamente en la prevención del consumo de tabaco por primera vez.

El tabaquismo continúa siendo un problema de salud alarmante, ya que se presenta a edades más tempranas reflejándose así en la población en estudio.

Un lugar idóneo para la aplicación de este tipo de apoyos lo constituye la escuela, reforzando así su papel de factor de protección.

\section{Bibliografía}

1. Organización Mundial de la Salud. La epidemia del tabaquismo mata cada año a 6 millones de personas. Disponible en:

http://www.who.int/features/factfiles/tobacco epidemic_facts/es/index.html. Consultado el15 de octubre 2010.

2. México. Programa contra el tabaquismo actualización 2011-2012. Disponible en:http://www.insp. $\mathrm{mx} /$ tabaco/Datoscontenido/Tabaquismo.pdf Consultado el 30 enero 2012
2. Tapia R, Kuri P, Gutiérrez M. Panorama epidemiológico del tabaquismo en México. Salud pública Méx [revista en la Internet]. 2001 Oct [citado 2013 Mayo 14] ; 43(5): 478-484. Disponible en:

http://www.scielo.org.mx/scielo.php?script $=$ sci arttext\&pid $=$ S0036-36342001000500012\&lng $=\mathrm{es}^{-}$. Consultado el 30 de enero 2012

3. Iglesias E. Bases Teóricas que sustentan los Programas de Prevención de Drogas. 40-41.

4. México. Comisión Nacional contra las Adicciones. Encuesta Nacional de Adicciones ENA 2011. Disponible en:http://www.conadic.salud.gob.mx/pdfs/ 
ENA 2011 DROGAS ILICITAS .pdf Consultado el 15 octubre de 2012

5. Orem E. D. Modelo de Orem. Conceptos de enfermería en la práctica. España. Ediciones Científicas y Técnicas S.A. España. Mc Graw Hill Interamericana. 1993. 74,152-153

6. Marriner T. Raire A. Modelos y Teorías en Enfermería. España. Harcourt. 1998. 23, 177-182.

7. Benavent A. Ferrer E. Fundamentos de enfermería. España. DAE. 2001. 315-344

8. Ostiguin R. Velazquez S. Teoría general del déficit del autocuidado. México. Manual Moderno. 2001 68

9. Wesley R. Teorías y Modelos de enfermería. 2a edición. México: Mc Graw Hill Interamericana. 1997. 90

10. American Health Association. Differences in worldwide tobacco use by gender: findings from the Global Youth Tobacco Survey. J School Health 2003. 207-209.

11. Hunt $M K$, Fagan $P$, Lederman $R$, Stoddard $A$ et al. Feasibility of implementing intervention methods in a adolescent worksite tobacco control study. Tobacco Control 2003. 40-46.

12. Tobler NS, Stratton HH. Effectiveness of school-based drug preventions programs: a meta-analysis of research. J Primary Prevention 1997. 18:71-28

13. Martínez L. Centre Cochrane Iberoamericano. Curso de introducción a la metodología de la investigación (II) Tipos de diseños a la investigación. [revista en la Internet]. 8 febrero 2011 [citado marzo 2011] Dis- ponible en: http://www.uv.es/invsalud/invsalud/ disenyo-tipo-estudio.htm\#disestudio

14. American Health Asociation. Instrumentos de medición tabaco test Fagerstrom. [Consultado el 30 enero 2012] Disponible en: http://www.uv.es/lisis/instrumentos09mexic.htm\#tres

15. México. Instituto Nacional de Geografía y Estadística. Estadísticas a Propósito del día mundial sin tabaco. Disponible en: http://www.inegi.org.mx/inegi/ default.aspx?s=inegi $\& c=2760 \& e=03 \&$ pred $=1$. Consultado el 5 de Octubre de 2010.

16. México. Comisión Nacional contra las Adicciones. Encuesta Nacional de Adicciones 2008. Disponible en: http://www.conadic.salud.gob.mx/pdfs/ena08/ ENA08_NACIONAL.pdf Consultado el 6 junio 2010.

17. Rodríguez-Ramírez José Manuel, Macías-Galaviz Ma. Tereza, Serna-Silva Carolina, Mendez-Navarrete Gabriela, Gómez-Cardona Juán Pablo, RodríguezNava, Victor Federico. Prevalencia de consumo de tabaco en estudiantes de bachillerato. Lux Médica (revista UAA) 2012 May-Ago (citado Ago 2012); 7(21): 3-8.

18. Valdés-Salgado, R., Thrasher, J., Sánchez-Zamorano, L.M., Lazcano-Ponce, E., Reynales-Shigematsu, L.M., Meneses-González, F. y Hernández-Ávila, M. (2006). Los retos del convenio marco para el control del tabaco en México: un diagnóstico a partir de la Encuesta sobre Tabaquismo en Jóvenes. En Salud Pública de México. Volumen 48. Suplemento 1. p. S5-S16. 